\title{
Strength training prior to muscle injury potentiates low-level laser therapy (LLLT)-induced muscle regeneration
}

\author{
Samuel Rodrigues Lourenço Morais ${ }^{1,2}$ • Alexandre Ginei Goya ${ }^{3}$ - Úrsula Urias ${ }^{4}$. \\ Paulo Roberto Jannig ${ }^{4}$ - Aline Villa Nova Bacurau ${ }^{4}$ - Wagner Garcez Mello ${ }^{1,2}$ • \\ Paula Lazilha Faleiros ${ }^{5}$ - Sandra Helena Penha Oliveira ${ }^{3}$ - Valdir Gouveia Garcia ${ }^{5}$. \\ Edilson Ervolino $^{3}$ - Patricia Chakur Brum ${ }^{4,6}$ - Rita Cássia Menegati Dornelles ${ }^{1,2,3}$
}

Received: 27 May 2016 / Accepted: 22 November 2016/Published online: 1 December 2016

(C) Springer-Verlag London 2016

\begin{abstract}
We evaluated whether strength training (ST) performed prior to skeletal muscle cryolesion would act as a preconditioning, improving skeletal muscle regeneration and responsiveness to low-level laser therapy (LLLT). Wistar rats were randomly assigned into non-exercised (NE), NE plus muscle lesion (NE + LE), NE + LE plus LLLT $(\mathrm{NE}+\mathrm{LE}+$ LLLT), strength training (ST), ST + LE, and ST + LE + LLLT. The animals performed 10 weeks of ST (climbing ladder; $3 \times$ week; $80 \%$ overload). Forty-eight hours after the last ST session, tibialis anterior (TA) cryolesion was induced and LLLT (InGaAlP, $660 \mathrm{~nm}, 0.035 \mathrm{~W}, 4.9 \mathrm{~J} / \mathrm{cm}^{2} /$ point, 3 points, spot light $0.028 \mathrm{~cm}^{2}, 14 \mathrm{~J} / \mathrm{cm}^{2}$ ) initiated and conducted daily for 14 consecutive days. The difference between intergroups was assessed using Student's $t$ test and intragroups by twoway analysis of variance. Cryolesion induced massive muscle degeneration associated with inflammatory infiltrate. Prior ST
\end{abstract}

Samuel Rodrigues Lourenço Morais

samuelrodrigues@foa.unesp.br

1 Programa Multicêntrico de Pós-Graduação em Ciências Fisiológicas-SBFis/UNESP, Araçatuba, Brazil

2 Laboratory of Endocrine Physiology and Aging-Department of Basic Sciences, Araçatuba, São Paulo, Brazil

3 São Paulo State University (UNESP), School of Dentistry, Araçatuba, São Paulo, Brazil

4 School of Physical Education and Sport, University of Sao Paulo, Sao Paulo, Brazil

5 Group of Research and Study on Laser in Dentistry (GEPLO), Division of Periodontics, Department of Surgery and Integrated Clinic, São Paulo State University (UNESP), School of Dentistry, Araçatuba, São Paulo, Brazil

6 Experimental Physiopathology-Medical School, University of Sao Paulo, Sao Paulo, Brazil improved skeletal regeneration 14-days after cryolesion and potentiated the regenerative response to LLLT. Cryolesion induced increased TNF- $\alpha$ levels in both NE + LE and ST + LE groups. Both isolated ST and LLLT reduced TNF- $\alpha$ to control group levels; however, prior ST potentiated LLLT response. Both isolated ST and LLLT increased IL-10 levels with no additional effect. In contrast, increased TA IL-6 levels were restricted to ST and $\mathrm{ST}+\mathrm{LE}+\mathrm{LLLT}$ groups. TA myogenin mRNA levels were not changed by neither prior ST or ST + LLLT. Both prior ST and LLLT therapies increased MyoD mRNA levels and, interestingly, combined therapies potentiated this response. Myf5 mRNA levels were increased only in ST groups. Taken together, our data provides evidences for prior ST potentiating LLLT efficacy in promoting skeletal muscle regeneration.

Keywords Skeletal muscle regeneration - Low-level laser therapy $\cdot$ Strength training $\cdot$ MyoD $\cdot$ Myogenin $\cdot$ Myf5

\section{Introduction}

Skeletal muscle lesions are common during sports practice both among professional and amateur practitioners $[1,2]$ and usually result in compromised muscle functionality. The muscle regenerative process is a complex phenomenon that involves several cellular and molecular signaling characterized by five interrelated and time-dependent phases: degeneration, inflammation, regeneration, remodeling, and maturation [3].

The inflammatory response is required and is crucial for a positive outcome of skeletal muscle regeneration. The neutrophils, macrophages and cytokines released by the muscle fibers during this phase exert essential function on skeletal muscle regeneration. Tumor necrosis factor alpha (TNF- $\alpha)$ is one of the key mediators of muscle inflammation and plays essential role in the cytokine cascade (i.e. IL-6 and IL-10) [4-9] 
which in turn influences simultaneously other steps of muscle regeneration. Concomitantly, the activation and differentiation of satellite cells (SC) occur that regulates in part the myogenic regulatory factors (MRFs). The MRFs exert essential role in skeletal muscle regeneration, whereas $\mathrm{MyoD}[10,11]$ and Myf5 act on the activity and proliferation of SC [12] and then myogenin is required for terminal differentiation of myotubes [13].

Among the several strategies to improve the skeletal muscle regeneration process, the low-level laser therapy (LLLT) has been elucidated as an effective therapeutic intervention on skeletal muscle injuries by reducing inflammatory mediators (TNF- $\alpha$, IL-6, and IL1- $\beta$ ) [14] and accelerating the muscle regeneration process $[1,15-17]$. In fact, the efficiency of LLLT on muscle regeneration is clear; however, how exactly the skeletal muscle mechanisms are influenced by LLLT during muscle regeneration process is not all clear.

Further to improving skeletal muscle regeneration, a growing body of studies has been demonstrating positive effects of LLLT applied prior and post exercise sessions related to muscle recovery [18-21]. Further, a previous study evaluated the effect of chronic aerobic exercise practice and LLLT interaction applied post a musculoskeletal atrophy model, verifying that the interaction with exercise and LLLT was effective in preventing the muscle atrophy [22]. However, to the best of our knowledge, we observed no studies aimed to evaluate the effects of strength training (ST) practice prior to skeletal muscle lesions and the interaction with LLLT in skeletal muscle regeneration.

It is indeed clear that chronic ST practice results in muscle hypertrophy, increased strength, higher myofibrillar content [23], as well as in the improvement in the anti-inflammatory system [24] and cytoprotective to muscle cells [25]. We hypothesize that chronic ST practice prior to skeletal muscleinduced lesion and the interaction with LLLT might improve skeletal muscle regeneration.

Therefore, the aim of the present study was to evaluate whether the ST practice prior to cryolesion-induced muscle lesion would improve the skeletal muscle regenerative process and the responsiveness to LLLT. For that, we assessed the skeletal muscle histology, pro- and anti-inflammatory markers, and myogenic regulator factors.

\section{Materials and methods}

\section{Animals and experimental groups}

Male Wistar rats weighing $350 \mathrm{~g}$ (4 months old) were obtained from the São Paulo State University (UNESP), School of Dentistry, Araçatuba, SP, Brazil. They were kept in a facility under controlled temperature $\left(2{ }^{\circ} \mathrm{C} \pm 2{ }^{\circ} \mathrm{C}\right)$ with $12: 12 \mathrm{~h}$ of light/dark cycle. Four animals were housed in each cage with free access to standard laboratory chow (Presence Ratos e Camundongos, Presence ${ }^{\circledR}$ S/A, Paulínia, SP, Brazil) and tap water. Forty-two Wistar male rats were randomly assigned into six groups with 7 animals per group: (1) non-exercised control group (NE), (2) NE plus muscle cryolesion (NE + LE), (3) NE + LE treated with LLLT (NE + LE + LLLT), (4) strength training control group (ST), (5) ST plus muscle cryolesion (ST + LE), and (6) ST + LE treated with LLLT $(\mathrm{ST}+\mathrm{LE}+\mathrm{LLLT})$ (Fig. 1).

\section{Strength training protocol and maximum voluntary carrying capacity (MVCC)}

The animals were familiarized with a climbing ladder $(1.1 \times$ $0.18 \mathrm{~m}, 2$-cm grid, $80^{\circ}$ incline-Engineered by Department of maintenance - São Paulo State University (UNESP), School of Dentistry, Araçatuba, SP, Brazill) for 3 days, based on previous studies $[23,26]$. The adaptation period was composed by 3 sessions, in non-consecutive days, without load. After that, the MVCC was obtained. A load apparatus ((plastic tube (BD Falcon $50 \mathrm{~mL}$ conical tube, BD Biosciences ${ }^{\circledR}$, Bedford, MA, USA)) containing steel balls (Esferas de aço, Cabana

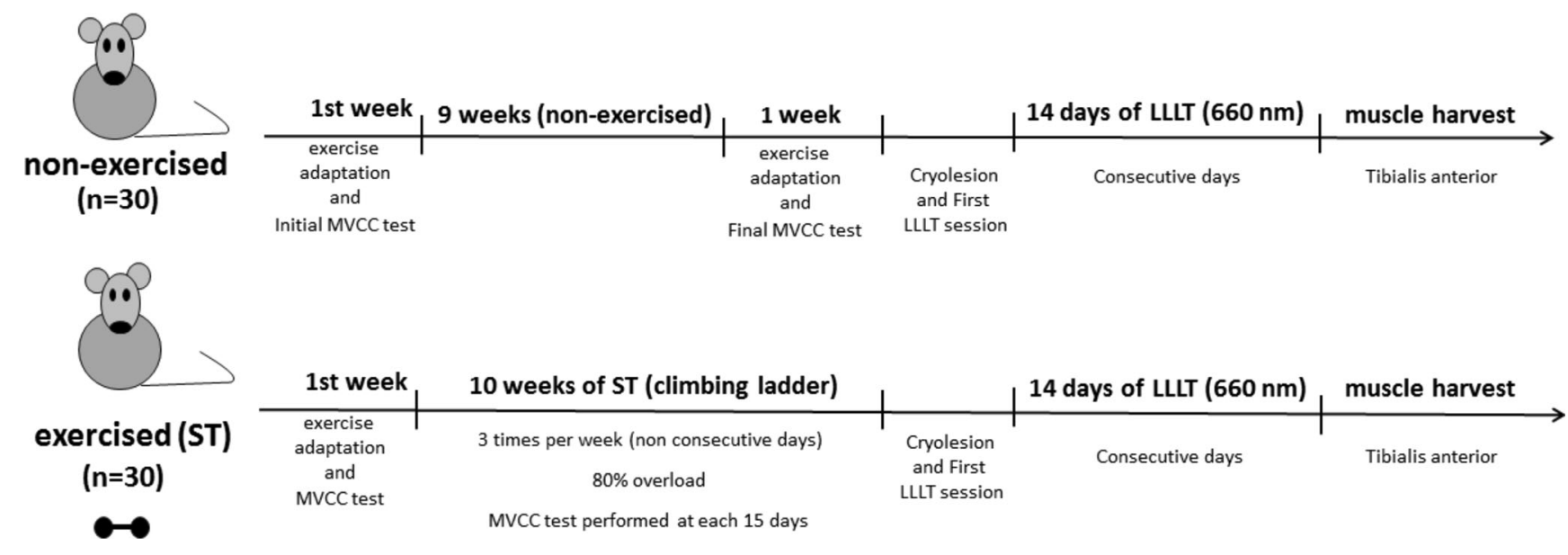

Fig. 1 Experimental design 
S/A, SP, Brazil) corresponding to $75 \%$ of the body mass as secured to the proximal portion of the rat's tail with a selfadhesive foam strip (Missner, Missner \& Missner Ltda, Blumenau, SC, Brazil). The rats performed repeated climbs with the load apparatus, resting $5 \mathrm{~min}$ each one climb. Then, $30 \mathrm{~g}$ of load was added to the load apparatus, and a new repetition was performed until climbing failure. The value of the load previous to failure climbing was considered the MVCC. The test was performed every 15 days with the aim to apply the correct load on each animal. The ST protocol consisted of 3 sessions per week (nonconsecutive days) for 10 weeks. Each session was composed of 6 sets of climbs, amounting to 48 and 62 dynamic movements (isotonic) per series [23]. The first week of the ST protocol began with an overload corresponding to $60 \%$ of the MVCC, increased to $70 \%$ in the second week and to $80 \%$ in the third week. The $80 \%$ of MVCC was maintained until the end of the protocol.

\section{Skeletal muscle injury (cryolesion)}

After 10 weeks of ST, the surgical cryolesion procedure was performed as previously described [27]. Briefly, the animals were deeply anesthetized (10-mg/kg body weight [bw] intraperitoneal [ip] xylazine (Coopazine ${ }^{\circledR}$, Coopers Brazil Ltda, Porto Feliz, SP, Brazil), and $80-\mathrm{mg} / \mathrm{kg}$ bw ip ketamine (Vetaset $^{\circledR}$, Fort Dodge Saude Animal Ltda, Porto Feliz, SP, Brazil), and the skin around the tibialis anterior (TA) muscle area was shaved and cleaned with Polyvinylpyrrolidone iodide (Indústria Química e Farmacêutica Rioquímica Ltda, Sao Jose do Rio Preto, SP, Brazil). Then, a transverse incision $(1 \mathrm{~cm})$ of the skin over in the middle of the TA muscle was created using a surgical blade (Solidor, Lamedid Comercial e Serviços Ltda, Barueri, SP, Brazil). The fascia was carefully removed, and the center of the TA muscles was surgically exposed. A hexagonal iron bar $(4.5 \mathrm{~mm})$ (Hexagonal key (Allen), Gedore, Sao Leopoldo, RS, Brazil), previously cooled for $10 \mathrm{~s}$ in liquid nitrogen (Agropercuaria R. L. Gonçalves - ME, Araçatuba, SP, Brazil), was kept for $10 \mathrm{~s}$ on the center of the TA muscles. This procedure was performed twice with an interval of $30 \mathrm{~s}$ of each one. The surgical surface was closed with a silk suture (3-0; SHALON Suturas $^{\circledR}$, Sao Luis de Montes Belos, GO, Brazil) and the animals received postsurgical intramuscular injections of 24,000 IU of penicillin G-benzathine (Pentabiótico Veterinário Pequeno Porte, Fort Dodge Saúde Animal Ltda, Campinas, SP, Brazil). After that, the animals subjected to cryolesion received the first LLLT session. Then, one animal was kept in each cage for a few hours on a warm box $\left(37^{\circ} \mathrm{C}\right)$ to prevent hypothermia. The TA muscle was analyzed due the fact to be constituted meanly by glycolitic fibers, mainly muscle fiber type actioned during ST practice, and to be one of the main muscles recruited during climbing ladder [28].

\section{Low-level laser therapy protocol}

An indium-gallium-aluminum phosphide (InGaAlP) laser (TheraLase $\AA$, DMC Equipamentos Ltda, Sao Carlos, SP, Brazil) with a wavelength of $660 \mathrm{~nm}$ was used in this study. Immediately after skin suturing, the first LLLT session was performed at 3 different points: the cryolesion site, $2 \mathrm{~mm}$ to the right of the cryolesion site, and $2 \mathrm{~mm}$ to the left of the cryolesion site. The therapeutic laser was applied at punctual contact with $660 \mathrm{~nm}, 0.035 \mathrm{~W}$, spot light $0.028 \mathrm{~cm}^{2}$, energy density of $4.9 \mathrm{~J} / \mathrm{cm}^{2} /$ point. The total energy density applied by day was $14.7 \mathrm{~J} / \mathrm{cm}^{2}$. The LLLT was performed daily at the same time for 14 consecutive days. The first application was applied by contact technique, with the optical fiber kept perpendicular to the skin immediately after skin suturing. The total energy density applied in the injured area after 14 consecutive days of LLLT therapy was $205.8 \mathrm{~J} / \mathrm{cm}^{2}$. The dose and parameters were based on a previous studies [29, 30]. In addition, we assessed the muscle regenerative process at 14 days post lesion and LLLT due to the fact that is a phase that precedes the total muscle recovery. Thus, improvements in this specific period could be positive signals for muscle regeneration.

\section{Skeletal muscle sampling}

Fourteen days after cryolesion induction and LLLT, the animals were anesthetized $(10-\mathrm{mg} / \mathrm{kg}$ bw intramuscular [im] xylazine (Coopazine ${ }^{\circledR}$, Coopers Brazil Ltda, Porto Feliz, SP, Brazil) and $80-\mathrm{mg} / \mathrm{kg}$ bw ip ketamine (Vetaset ${ }^{\circledR}$, Fort Dodge Saude Animal Ltda, Porto Feliz, SP, Brazil). The TA muscles were carefully harvested and weighed. The right TA was used for histopathological evaluation (hematoxylin-eosin [H\&E], Erviegas Ltda, SP, Brazil), and the left TA was used for ELISA (R\&D Systems, Minneapolis, MN, USA) and qRTPCR analyses (StepOnePlus, Applied Biosystems ${ }^{\circledR}$, Foster, CA, USA). The right TA was mounted in an optimal cutting temperature compound (Tissue-Tek, Sakura Finetek Inc, Torrance, CA, USA), flash-frozen in isopentane (2Methylbutane, Sigma ${ }^{\circledR}$, Saint Louis, MO, USA), and stored at $-80{ }^{\circ} \mathrm{C}$ (Indrel Ultra Freezer, Indrel Ind Ltda, PR, Brazil). The left TA was snap frozen in liquid nitrogen and stored at $-80{ }^{\circ} \mathrm{C}$ for later analyses.

\section{Histopathological evaluation}

The TA muscle was serially cut into $10-\mu \mathrm{m}$-thick sections by using a cryostat (Leica CM1850, Leica Biosystems, Nussloch, BW, Germany) across the central region of the muscle injury. The sections were cut longitudinally based in previous reports [31-33]. The tissue specimens were routinely processed, and H\&E staining was performed. A certified histologist (EE) blinded to the treatments performed the histological analysis. 
The following parameters were evaluated: nature and degree of inflammation, extent of the inflammatory process, presence and extent of tissue necrosis, vasculature status, structure pattern of the muscle and connective tissue, cellular pattern of the muscle and connective tissue, and the incidences of hyperplasia, metaplasia, and dysplasia.

\section{Skeletal muscle cytokine measurement}

The TA muscle tissue were homogenized in PBS (SigmaAldrich, Saint Louis, Missouri, USA) supplemented with protease inhibitors (Protease Inhibitor Cocktail, Sigma-Aldrich, Saint Louis, MS, USA) by using a Polytron homogenizer (KINEMATICA Polytron ${ }^{\mathrm{TM}}$ PT 1300D, Kinematica AG, Bohemia, NY, USA) and centrifuged $(1811 \times g ; 10 \mathrm{~min}$; $4{ }^{\circ} \mathrm{C}$ ). The supernatant was collected and stored at $-80{ }^{\circ} \mathrm{C}$ until use. A 96-well plate (Coastar, Corning ${ }^{\mathrm{TM}}$, New York, NY, USA) was coated with monoclonal antibody to TNF- $\alpha$ (R\&D Systems, Cat number \#BAF540, Minneapolis, MN, USA), IL-6 (R\&D Systems, Cat number \#MAB506, Minneapolis, MN, USA), and IL-10 (R\&D Systems, Cat number \#MAB519, Minneapolis, MN, USA). Samples or recombinant standards were added to the wells. After $2 \mathrm{~h}$, unbound proteins were washed away, and an enzyme-linked polyclonal antibody was added to the wells. This antibody acted as a link between TNF- $\alpha$, IL- 6 , and IL-10, and a dye agent. The color changes proportional to the amount of TNF- $\alpha$, IL-6, and IL-10 was observed. This was quantified by comparing the optical densities at $450 \mathrm{~nm}$ of the samples with its respective recombinant standard curve of known dilutions by using a plate reader at $450 \mathrm{~nm}$. The TNF- $\alpha$, IL- 6 , and IL-10 concentrations were calculated from a standard curve in $\mathrm{pg} / \mathrm{mL}$.

\section{Total RNA extraction and cDNA synthesis}

Total RNA was extracted from frozen TA muscle using the TRIzol $^{\circledR}$ reagent (Life Technologies, Carlsbad, CA, USA) according to manufacturer's instructions. An evaluation of the total RNA concentration and purity was performed by measuring the absorbance at 260 and $280 \mathrm{~nm}$ using NanoDrop Spectrophotometer (NanoDrop 1000 Spectrophotometer, Thermo Scientific, Wilmington, DE, USA), and 260/280 ratios ranging from 1.8 to 2.0 were considered satisfactory for purity standards. Denaturing agarose gel electrophoresis was used to assess the quality of the samples. A conventional reverse transcription reaction was performed to yield singlestranded cDNA. First-strand cDNA was synthesized from $1 \mu \mathrm{g}$ of total RNA using High Capacity RNA-to-cDNA kit according to the manufacturer's recommendations (Applied Biosystems, Carlsbad, CA, USA). The resulting cDNA was stored at $-20{ }^{\circ} \mathrm{C}$ until the expression analysis.

\section{Quantitative real-time PCR (qRT-PCR)}

The relative expression levels of selected genes in sample tissues were analyzed by qRT-PCR; the assays were performed in triplicate using SYBR Green I with an ABI 7500 Real-time PCR System (Applied Biosystems, Carlsbad, CA, USA). The genes analyzed were $\mathrm{MyoD}$ and myogenin. The relative expression data were calculated based on the geometric mean of cyclophilin as a housekeeping gene. All primers were designed based on different exons to avoid DNA amplification and were synthesized by Exxtend (Exxtend Ltda, Campinas, SP, Brazil). The specific primer sequences used for qRT-PCR were: MyoD (sense: 5' - TTC AGA TGG CGA ACT TGT CAG A - 3'; antisense: 5' - GGC CAC CAT TCT GTA TCT TGC T - 3'), myogenin (sense: 5' - GGT CCC AAC CCA GGA GAT CA 3'; antisense: 5' - GCT GTC CAC GAT GGA CGT AA - 3'), Myf5 (sense: 5' - CTC CAA CTG CTC TGA TGG CA - 3'; antisense: 5' - ATC CAA GCT GGA CAA GGA GC - 3') and Cyclophylin (sense: 5' - TGG CAA GCA TGT GGT TCT TTG GGA AG - 3'; antisense: 5' - GGT GAT CTT CTT GCT GGT CTT GCC ATT C - 3'). SYBR Green I amplification mixtures $(10 \mu \mathrm{L})$ contained 2 ng of cDNA, $5 \mu \mathrm{L}$ of $2 X$ SYBR Green I Master Mix (Life Technologies, Carlsbad, CA, USA), and forward and reverse primers. The cycle conditions included incubation at $50{ }^{\circ} \mathrm{C}$ for $5 \mathrm{~min}$, initial denaturation at $95^{\circ} \mathrm{C}$ for $10 \mathrm{~min}$, and 40 cycles at $95^{\circ} \mathrm{C}$ for $15 \mathrm{~s}$ and $60{ }^{\circ} \mathrm{C}$ for $1 \mathrm{~min}$. A DNA melting curve analysis showed a single peak for all genes. Results were expressed using the comparative cycle threshold (Ct) method [34]. The $\Delta \mathrm{Ct}$ obtained from the subtraction of target gene and the reference gene (cyclophylin) Ct's was used to calculate de $\Delta \Delta \mathrm{Ct}$ from other groups in relation to control group. The relative expression values were calculated with the equation $2-\Delta \Delta \mathrm{Ct}$.

\section{Statistical analysis}

All data obtained were presented as mean \pm standard error of mean (SEM). Shapiro-Wilks test was performed to verify normal distribution of the data. Difference between the intergroups were assessed using Student's $t$ test (for MVCC evaluation) and for intragroups using two-way analysis of variance (ANOVA) (GraphPad Prism 6.0, La Jolla, CA, USA) followed by Bonferroni post-hoc tests to identify significant differences between two variables in multiple groups. The data presented in qRT-PCR was performed on at least seven different samples/animals in biological triplicates. Sample size was predetermined based on the variability observed in preliminary and similar experiments. For all analyses, $p \leq 0.05$ was considered statistically significant. The statistical study power was estimated at least $80 \%$ ( $\beta$ factor $=20 \%$ ) (openepi software, Open Source Epidemiologic Statistics for Public Health, www.OpenEpi.com). 


\section{Results}

\section{Maximal voluntary carrying capacity (MVCC)}

To verify whether the chronic ST protocol used in the present study was effective, we evaluated the animal's MVCC. As shown in Fig. 2, the MVCC was similar in the beginning of ST protocol among the groups. However, ST animals displayed a progressive MVCC increase capacity during the experimental protocol compared to non-exercised animals.

\section{Histopathological evaluation of skeletal muscle regeneration}

In order to evaluate whether ST perform prior to muscleinduced lesion and the combined therapies applied post muscle cryolesion could improve the skeletal muscle regeneration, we assessed the TA muscle histological feature. The perimysium and endomysium of the NE and ST groups consisted of moderately dense connective and flaccid tissues, respectively, without much distinction between the groups (Fig. 3a, b). The lesioned animals without LLLT (NE + LE and ST + LE) displayed very similar histological features (Fig. 3c, d). The structural integrity of the muscle cells was either totally or partially lost. These groups showed extensive interstitial edema, intense inflammatory infiltration composed predominantly by neutrophils and lymphocytes, and many muscle fibers in an advanced stage of degeneration. However, the area of muscle fiber degeneration was smaller in the ST + LE group than in the NE + LE group (Fig. 3d). The groups that received LLLT (NE + LE + LLLT and ST + LE + LLLT) presented higher structural integrity of the endomysium, and especially the perimysium, than those who did not receive LLLT (NE + LE and ST + LE) (Fig. 3e, f). The amount of interstitial edema was lower, the areas of inflammatory infiltration and

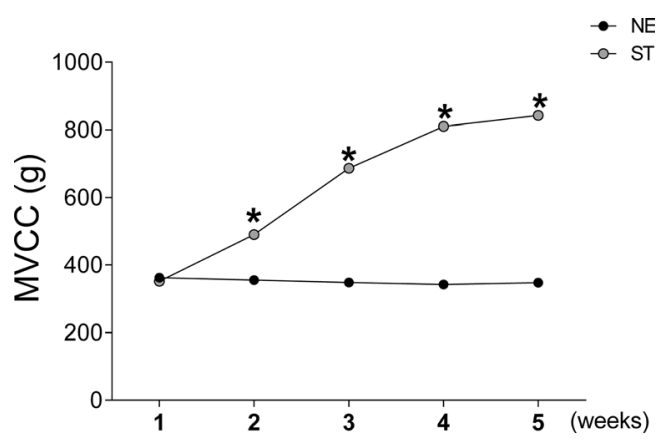

Fig. 2 Maximal voluntary carrying capacity (MVCC). Progressive MVCC tests performed during 10 weeks of strength training (ST)training (climbing ladder). The MVCC tests were performed. 1 (first test - after exercise adaptation), 2 (after 15 days), 3 (after 30 days), 4 (after 45 days), and 5 (performed 1 week before to the end of experimental protocol). The animals were randomly assigned into nonexercised control group (NE, $n=7$ ) and ST control group (ST, $n=7$ ). Values are presented as mean \pm SEM. $* p<0.05$ using the Student's $t$ test
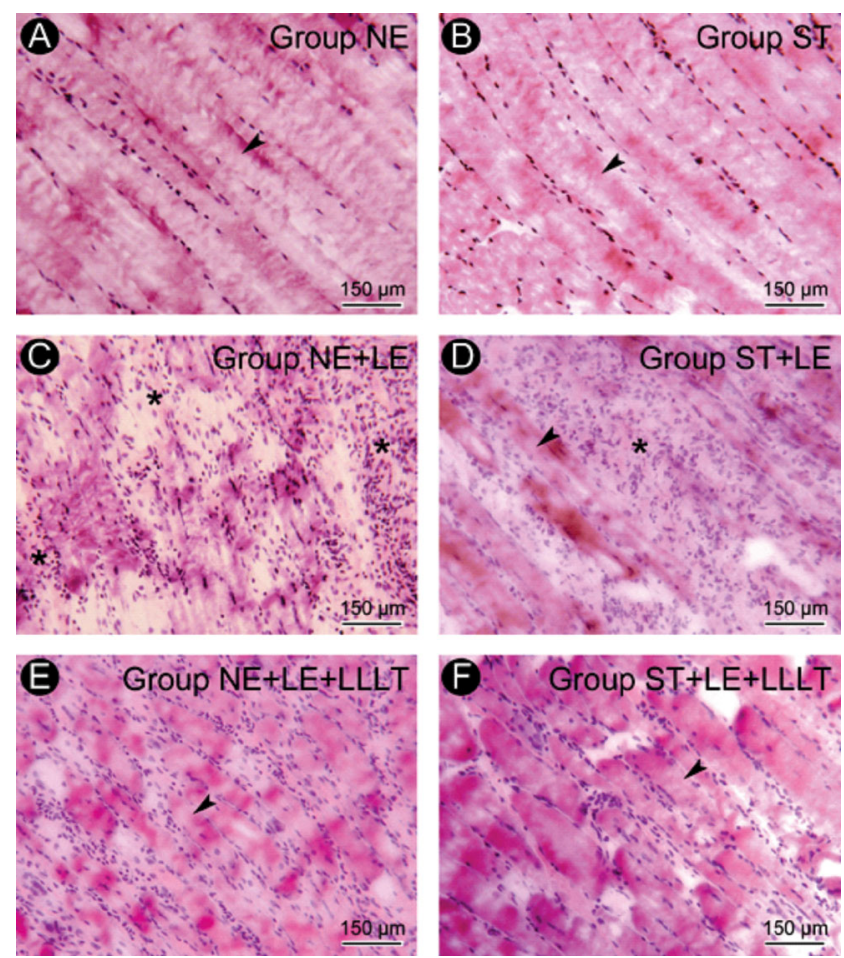

Fig. 3 Histopathological evaluation from skeletal muscle. Representative tibialis anterior (TA) muscle longitudinal sections dye with hematoxylin and eosin (H\&E). The histological feature from uninjured muscle of non-exercised (NE) (a) and strength-training exercised (ST) groups (b). The histological appearance of cryolesioned area in TA muscle from NE plus lesion (NE + LE) (c) (plus lesion) and ST + LE groups (d) groups, which were not subjected to laser irradiation, and the NE + LE + LLLT (e) and ST + LE + LLLT (f) groups, which were treated with LLLT (InGaAlP, $660 \mathrm{~nm}, 0.035 \mathrm{~W}, 4.9 \mathrm{~J} / \mathrm{cm}^{2} /$ point, 3 points $\left.(12 \mathrm{~s}), 14 \mathrm{~J} / \mathrm{cm}^{2}\right)$. Is possible to observe more advanced TA muscle regeneration in both groups treated with LLLT, especially with combined therapies. Experimental groups were composed by 7 animals each one. Symbols: arrows, muscle fiber; asterisks, area of loose connective tissue with fibroblasts and inflammatory cells. Original magnification: $\times 160$. Scale bar $150 \mu \mathrm{m}$

degenerated muscle fiber were reduced in comparison to those in the NE + LE and ST + LE groups. The ST + LE + LLLT group showed remarkable improvement with respect to these parameters and presented significant areas of granulation tissue, and better rearrangement of muscle fibers, reflecting in a more-rapid repair process in the exercised groups that were subject to cryolesion and treated with LLLT (Fig. 3e, f).

\section{Skeletal muscle cytokines levels}

It is well recognized that TNF- $\alpha$, IL-6, and IL-10 play important role in the inflammatory response and in muscle repair process $[35,36]$. Accordingly, we investigated TNF- $\alpha$, IL-6, and IL-10 levels from TA muscle. We did not observe difference in TNF- $\alpha$ levels among NE and ST animals (Fig. 4a), however, when lesioned, both groups exhibited significant increase of TNF- $\alpha$ levels (Fig. 4a). When treated with LLLT (NE + LE + LLLT and ST + 


\section{Control $\square$ Lesioned $\square$ Lesioned+LLLT}
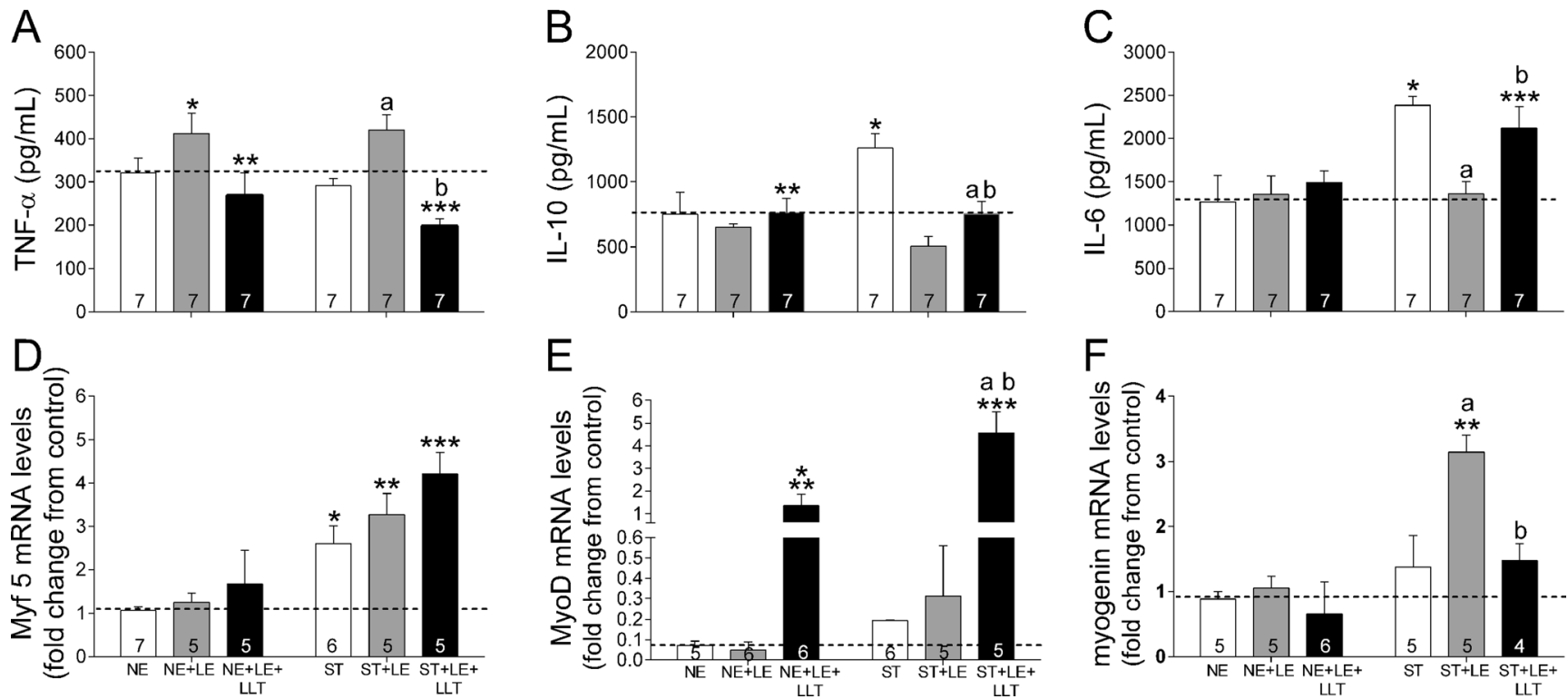

Fig. 4 Skeletal muscle pro/anti-inflammatory and mRNA levels of MRFs. Tibialis anterior (TA) muscle levels of TNF- $\alpha$ (a), IL-10 (b), IL-6 (c) and mRNA levels of Myf5 (d), Myf5 (e) and myogenin (F) after 14 days of cryolesion and LLLT therapy [InGaAlP, $660 \mathrm{~nm}, 0.035 \mathrm{~W}$, $4.9 \mathrm{~J} / \mathrm{cm}^{2} /$ point, 3 points ( $12 \mathrm{~s}$ ), $14 \mathrm{~J} / \mathrm{cm}^{2}$ ] evaluated in animals randomly assigned into non-exercised control group (NE), NE plus muscle

cryolesion (NE + LE), NE + LE treated with LLLT (NE + LE + LLLT), strength training control group (ST), ST plus muscle lesion (ST + LE) and ST + LE treated with LLLT (ST + LE + LLLT). Values are presented as mean \pm SEM. * vs NE; **v vs NE + LE; *** vs NE + LE + LLLT; $a$ vs $\mathrm{ST} ; b$ vs ST $+\mathrm{LE}$ and using one-way ANOVA. The number of animals studied is indicated in bar graph representing each group

LE + LLLT), the animals displayed great reduction in TNF- $\alpha$ levels (Fig. 4a). However, combined therapies $(\mathrm{ST}+\mathrm{LE}+\mathrm{LLLT})$ potentiated this response compared to non-exercised animals (NE + LE + LLLT).

Regarding to TA IL-10, when lesioned, only exercised animals $(\mathrm{ST}+\mathrm{LE})$ displayed significant reduction in IL-10 levels in comparison to non-exercised and lesioned animals (NE + LE) (Fig. 4b). In addition, the IL-10 levels were significantly increased when the LLLT was applied in both groups, however, interestingly, the IL-10 levels were higher in exercised animals (ST + LE + LLLT) in comparison to nonexercised animals (NE + LE + LLLT) (Fig. 4b).

As observed in IL-10 levels, ST practice promoted significant increase in IL-6 levels in comparison to non-exercised animals (NE) (Fig. 4c). We observed no difference in IL-6 levels when the animals were lesioned and only the exercised animals were responsive to LLLT (ST + LE + LLLT), displaying significant increase in TA IL-6 levels (Fig. 4c).

\section{mRNA expression of MyoD, Myogenin, and Myf5}

In order to test whether ST practice could promote more distinct skeletal muscle response during muscle repair than those of nonexercised animals, we evaluated the messenger RNA (mRNA) levels of Myf5, MyoD, and myogenin. The exercised animals (ST), lesioned or not, displayed significant increase in Myf5 mRNA levels compared to non-exercised animals (NE)

(Fig. 4d). Further, only the exercised and lesioned animals (ST + LE + LLLT) displayed responsiveness to LLLT, resulting in significant increase in Myf5 mRNA levels (Fig. 4d). The muscle lesion did not alter MyoD mRNA levels; however, LLLT shown to be able to significantly increase MyoD mRNA levels in lesioned animals, exercised (ST + LE + LLLT) or not (NE + LE + LLLT) (Fig. 4e). Interestingly, combined therapies $(\mathrm{ST}+\mathrm{LE}+\mathrm{LLLT})$ potentiated LLLT response with great significance (Fig. 4e). Similarly, as observed in MyoD, prior ST practice did not alter the myogenin mRNA expression in comparison to non-exercised animals (NE) (Fig. 4e). However, when lesioned, the exercised animals $(\mathrm{ST}+\mathrm{LE})$ displayed great increase in myogenin mRNA levels compared to non-exercised animals $(\mathrm{NE}+\mathrm{LE})$ (Fig. 4f). The combined interaction resulted in significant reduction in myogenin levels while no differences were observed in NE + LE + LLLT group (Fig. 4f).

\section{Discussion}

In the present study, we demonstrated that ST performed previously to skeletal muscle cryoinjury combined with LLLT significantly improved the skeletal muscle regeneration. Interestingly, the exercised animals displayed more responsiveness to LLLT, attenuating skeletal muscle inflammatory markers, increasing MRFs levels resulting in more advanced muscle regeneration (Fig. 5). 
Fig. 5 Schematic illustration with the main results achieved in the present study

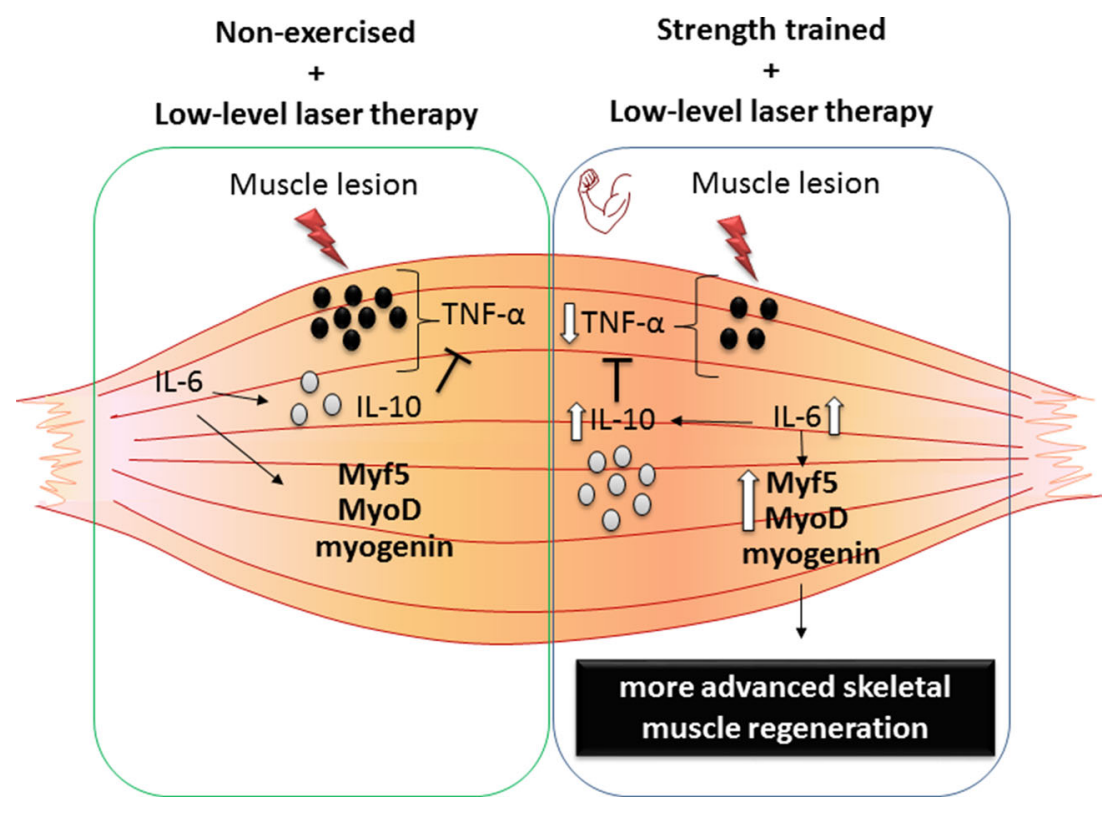

LLLT has been demonstrating efficiency not only improving and accelerating the skeletal muscle regeneration $[1,15$, 17] but also providing positive influence on physical performance and muscle recovery [19, 20, 37, 38]. In this sense, studies have been designed to evaluate the effects of combined therapies (LLLT and exercise practice) applied post muscleinduced injuries in skeletal muscle regenerative process [22, 39]. Assis et al. [19, 39] demonstrated that LLLT and aerobic exercise association applied post a muscle-induced atrophy model significantly reduced muscle RING-finger protein-1 and Atrogin-1 mRNA levels, preventing muscle atrophy.

Unlike other recent studies, we evaluated whether prior ST performed before skeletal muscle cryolesion would act as a precondition, improving skeletal muscle regeneration and responsiveness to LLLT. In fact, the combined therapies (ST and LLLT) improved the muscle regeneration process attenuating muscle inflammatory markers (assessed by muscle histology) and upregulating MRFs factors, potentiating the known LLLT positive effects related to muscle regenerative process.

Indeed, LLLT has been proven effective to resolve the inflammatory markers $[14,40]$. Interestingly, in our study, combined therapies potentiated this response (e.g., TNF- $\alpha$, IL-6), suggesting more responsiveness of exercised animals (ST + LE + LLLT) to LLLT compared to non-exercised animals (NE + LE + LLLT). In addition, ST practice isolated and in combination with LLLT promoted significant increase of IL-10 levels, supporting the hypothesis that chronic ST practice provides an improved antiinflammatory response [24]. The effects of ST and LLLT in IL10 mRNA levels might be mediated by IL-6, considering that chronic exercise practice-induced IL- 6 production is followed by increased circulating anti-inflammatory cytokines, such as IL-10 [41]. Further, decreased levels of TNF- $\alpha$ might be resultant of combined therapies-induced, increasing IL-10 levels. That, in turn, inhibits the TNF- $\alpha$ production [41], resulting in attenuated inflammatory levels in exercised animals.

Furthermore, recent studies have shown the important role of IL-6 in skeletal muscle regeneration, suggesting that this cytokine is involved in proliferation, differentiation and fusion of muscle cells [35, 41-43]. In addition, IL-6 seems to be directly involved in MRFs expression [35] that orchestrated muscle regeneration [3]. As observed in inflammatory markers levels, combined therapies promoted significant improvements in MRFs expression. In the present study, prior ST animals displayed higher Myf5 and myogenin mRNA levels when lesioned, suggesting a possible improvement on proliferation (Myf5) and terminal differentiation (myogenin) of muscle cells in these animals. Further, combined therapies seem to accelerate muscle regeneration by improving myogenesis, differentiation and terminal differentiation (e.g., MyoD and myogenin) of lesioned muscle cells, based on Myf5, MyoD and myogenin mRNA expression [3] as well histopathological results. Briefly, we suggest that prior ST practice-induced accumulates benefits previously to muscleinduced lesion, seemingly, potentiating the LLLT effects, improving skeletal muscle regeneration.

\section{Conclusion and summary}

Taken altogether, our results provide evidences for combined therapies (ST and LLLT) improving the skeletal muscle regeneration. These positive outcomes seem to be resultant of attenuated inflammation and also of a positive modulation of MRFs. Thus, prior ST performed previously to skeletal muscle-induced lesion in combination with LLLT may have therapeutic potential, especially in situations where the subject could be incapable to perform any type of physical exercise therapy or even to walk. 
Acknowledgements The authors would like to thank to Fundação de Amparo à Pesquisa do Estado de São Paulo (FAPESP) by the financial support and scholarship (FAPESP Grant n 2013/18907-2).

\section{Compliance with ethical standards}

Ethics statement All animal procedures were approved by the Institutional Animal Care and Use Committee of the Faculty of Dentistry (Univ. Estadual Paulista-UNESP, Araçatuba, Sao Paulo, Brazil) (ethical committee approval-process $n^{\circ}$. 2013/01543) and complied with guidelines of the Brazilian National Council for the Control of Animal Experimentation. The authors declare that this manuscript was prepared in accordance to the "Ethical Responsibilities of Authors" and it is an unpublished material which it is not being evaluated for publication in other journals. The manuscript has been read by all authors and the contributions performed by each one were approved. This study received financial support from the Fundação de Amparo à Pesquisa do Estado de São Paulo, Brazil (FAPESP Grant n 2013/18907-2).

Conflict of interest The authors declare no conflict of interest in this study.

\section{References}

1. Ribeiro BG, Alves AN, Santos LA, Fernandes KP, Cantero TM et al (2015) The effect of low-level laser therapy (LLLT) applied prior to muscle injury. Lasers Surg Med 47:571-78

2. de Melo CA, Alves AN, Terena SM, Fernandes KP, Nunes FD et al (2015) Light-emitting diode therapy increases collagen deposition during the repair process of skeletal muscle. Lasers Med Sci 31:531-8

3. Musarò A (2014) The Basis of Muscle Regeneration. Adv Biol 116

4. Fukuda TY, Tanji MM, Jesus JF, Sato NM, Duarte AJ et al (2010) Single session to infrared low level diode laser on TNF- $\alpha$ and IL-6 cytokines release by mononuclear spleen cells in mice: A pilot study. Lasers Surg Med 42:584-588

5. Pires D, Xavier M, Araújo T, Silva JA Jr, Aimbire F et al (2011) Low-level laser therapy (LLLT; $780 \mathrm{~nm}$ ) acts differently on mRNA expression of anti- and pro-inflammatory mediators in an experimental model of collagenase-induced tendinitis in rat. Lasers Med Sci 26:85-94

6. Dinarello CA (2000) Proinflammatory cytokines. Chest 118:503-508

7. Ostrowski K, Schjerling P, Pedersen BK (2000) Physical activity and plasma interleukin-6 in humans - effect of intensity of exercise. Eur J Appl Physiol 83:512-5

8. Ostrowski K, Rohde T, Asp S, Schjerling P, Pedersen BK (1999) Pro- and anti-inflammatory cytokine balance in strenuous exercise in humans. J Physiol 515:287-291

9. Cornelison DD, Olwin BB, Rudnicki MA, Wold BJ (2000) MyoD $(-/-)$ satellite cells in single-fiber culture are differentiation defective and MRF4 deficient. Dev Biol 224:122-137

10. Grounds MD, Garrett KL, Lai MC, Wright WE, Beilharz MW (1992) Identification of skeletal muscle precursor cells in vivo by use of MyoD1 and myogenin probes. Cell Tissue Res 267:99-104

11. Füchtbauer EM, Westphal H (1992) MyoD and myogenin are coexpressed in regenerating skeletal muscle of the mouse. Dev Dyn 193:34-9

12. Tidball JG, Wehling-Henricks M (2007) Macrophages promote muscle membrane repair and muscle fibre growth and regeneration during modified muscle loading in mice in vivo. J Physiol 578:327-336
13. Tidball JG, Villalta SA (2010) Regulatory interactions between muscle and the immune system during muscle regeneration. Am J Physiol Regul Integr Comp Physiol 298:R1173-87

14. Leal-Junior EC, de Almeida P, Tomazoni SS, de Carvalho PT, Lopes-Martins RÁ et al (2014) Superpulsed low-level laser therapy protects skeletal muscle of $\mathrm{mdx}$ mice against damage, inflammation and morphological changes delaying dystrophy progression. PLoS One 9, e89453

15. Alves AN, Fernandes KP, Deana AM, Bussadori SK, MesquitaFerrari RA (2014) Effects of low-level laser therapy on skeletal muscle repair: a systematic review. Am J Phys Med Rehabil 93: 1073-85

16. Baptista J, Martins MD, Pavesi VC, Bussadori SK, Fernandes KP et al (2011) Influence of laser photobiomodulation on collagen IV during skeletal muscle tissue remodeling after injury in rats. Photomed Laser Surg 29:11-7

17. Assis L, Moretti AI, Abrahão TB, de Souza HP, Hamblin MR et al (2013) Low-level laser therapy (808 $\mathrm{nm}$ ) contributes to muscle regeneration and prevents fibrosis in rat tibialis anterior muscle after cryolesion. Lasers Med Sci 28:947-955

18. Larkin-Kaiser KA, Christou E, Tillman M, George S, Borsa PA et al (2015) Near-infrared light therapy to attenuate strength loss after strenuous resistance exercise. J Athl Train 50:45-50

19. Assis L, Yamashita F, Magri AM, Fernandes KR, Yamauchi L et al (2015) Effect of low-level laser therapy (808 nm) on skeletal muscle after endurance exercise training in rats. Braz J Phys Ther 19:457-465

20. Patrocinio T, Sardim AC, Assis L, Fernandes KR, Rodrigues N et al (2013) Effect of low-level Laser therapy (808 nm) in skeletal muscle after resistance exercise training in rats. Photomed Laser Surg 31:492-498

21. Toma RL, Tucci HT, Antunes HK, Pedroni CR, de Oliveira AS et al (2013) Effect of $808 \mathrm{~nm}$ low-level laser therapy in exercise-induced skeletal muscle fatigue in elderly women. Lasers Med Sci 28:1375-82

22. Assis L, Almeida T, Milares LP, dos Passos N, Araújo B et al. (2015) Musculoskeletal Atrophy in an Experimental Model of Knee Osteoarthritis: The Effects of Exercise Training and LowLevel Laser Therapy. Am J Phys Med Rehabil 609-616

23. Hornberger TA Jr, Farrar RP (2004) Physiological hypertrophy of the FHL muscle following 8 weeks of progressive resistance exercise in the rat. Can J Appl Physiol 29:16-31

24. Alves JP, Nunes RB, Stefani GP, Dal Lago P (2014) Resistance training improves hemodynamic function, collagen deposition and inflammatory profiles: experimental model of heart failure. PLoS One 9, e110317

25. Wiggs MP (2015) Can endurance exercise preconditioning prevention disuse muscle atrophy? Front Physiol 6:63

26. Stringhetta-Garcia CT, Singulani MP, Santos LF, Louzada MJ, Nakamune AC et al (2016) The effects of strength training and raloxifene on bone health in aging ovariectomized rats. Bone 85:45-54

27. Miyabara EH, Martin JL, Griffin TM, Moriscot AS, Mestril R (2006) Overexpression of inducible 70-kDa heat shock protein in mouse attenuates skeletal muscle damage induced by cryolesioning. Am J Physiol Cell Physiol 290:C1128-38

28. Jung S, Ahn N, Kim S, Byun J, Joo Y et al (2015) The effect of ladderclimbing exercise on atrophy/hypertrophy- related myokine expression in middle-aged male Wistar rats. J Physiol Sci 65:515-521

29. Rodrigues NC, Brunelli R, de Araújo HS, Parizotto NA, Renno AC (2013) Low-level laser therapy (LLLT) $(660 \mathrm{~nm})$ alters gene expression during muscle healing in rats. J Photochem Photobiol B 120: 29-35

30. Fernandes KP, Alves AN, Nunes FD, Souza NH, Silva JA Jr et al (2012) Effect of photobiomodulation on expression of IL-1 $\beta$ in skeletal muscle following acute injury. Lasers Med Sci 28:1043-1046

31. Rennó AC, Toma RL, Feitosa SM, Fernandes K, Bossini PS et al (2011) Comparative effects of low-intensity pulsed ultrasound and 
low-level laser therapy on injured skeletal muscle. Photomed Laser Surg 29:5-10

32. Liu XG, Zhou YJ, Liu TC, Yuan JQ (2009) Effects of low-level laser irradiation on rat skeletal muscle injury after eccentric exercise. Photomed Laser Surg 27:863-869

33. Rodrigues NC, Brunelli R, de Araújo HS, Parizotto NA, Renno AC (2013) Low-level laser therapy (LLLT) $(660 \mathrm{~nm})$ alters gene expression during muscle healing in rats. J Photochem Photobiol B Biol 120:29-35

34. Livak KJ, Schmittgen TD (2001) Analysis of relative gene expression data using real-time quantitative PCR and the 2(-Delta Delta C(T)) Method. Methods 25:402-408

35. Alves AN, Ribeiro BG, Fernandes KP, Souza NH, Rocha LA et al (2016) Comparative effects of low-level laser therapy pre- and postinjury on mRNA expression of MyoD, myogenin, and IL-6 during the skeletal muscle repair. Lasers Med Sci 31:679-85

36. Hawke TJ, Garry DJ (2001) Myogenic satellite cells: physiology to molecular biology. J Appl Physiol (1985) 91:534-51

37. Ferraresi C, de Brito Oliveira T, de Zafalon Oliveira L, de Menezes Reiff RB, Baldissera Vet al (2011) Effects of low level laser therapy $(808 \mathrm{~nm})$ on physical strength training in humans. Lasers Med Sci $26: 349-58$
38. Leal-Junior EC, Vanin AA, Miranda EF, de Carvalho PT, Dal Corso $S$ et al (2015) Effect of phototherapy (low-level laser therapy and light-emitting diode therapy) on exercise performance and markers of exercise recovery: a systematic review with meta-analysis. Lasers Med Sci 30:925-939

39. Assis L, Milares LP, Almeida T, Tim C, Magri A et al (2016) Aerobic exercise training and low-level laser therapy modulate inflammatory response and degenerative process in an experimental model of knee osteoarthritis in rats. Osteoarthr Cartil 24:169-177

40. Assis L, Moretti AI, Abrahão TB, Cury V, Souza HP et al (2012) Low-level laser therapy $(808 \mathrm{~nm})$ reduces inflammatory response and oxidative stress in rat tibialis anterior muscle after cryolesion. Lasers Surg Med 44:726-735

41. Kurosaka M, Machida S (2013) Interleukin-6-induced satellite cell proliferation is regulated by induction of the JAK2/STAT3 signalling pathway through cyclin D1 targeting. Cell Prolif 46:365-373

42. Hoene M, Runge H, Häring HU, Schleicher ED, Weigert C (2013) Interleukin-6 promotes myogenic differentiation of mouse skeletal muscle cells: role of the STAT3 pathway. Am J Physiol Cell Physiol 304:C128-36

43. Pedersen BK (2007) IL-6 signalling in exercise and disease. Biochem Soc Trans 35:1295-1297 